\title{
Identification of the ventral occipital visual field maps in the
}

\section{human brain [version 1; peer review: 2 approved, 1 approved}

\section{with reservations]}

\author{
Jonathan Winawer(i)1, Nathan Witthoft ${ }^{2}$ \\ ${ }^{1}$ Psychology and Center for Neural Science, New York University, New York, NY, 10003, USA \\ ${ }^{2}$ Department of Psychology, Stanford University, Stanford, CA, 94305, USA
}

\author{
V1 First published: 21 Aug 2017, 6:1526 \\ https://doi.org/10.12688/f1000research.12364.1 \\ Latest published: 21 Aug 2017, 6:1526 \\ https://doi.org/10.12688/f1000research.12364.1
}

\section{Abstract}

The location and topography of the first three visual field maps in the human brain, V1-V3, are well agreed upon and routinely measured across most laboratories. The position of $4^{\text {th }}$ visual field map, ' $h V 4$ ', is identified with less consistency in the neuroimaging literature. Using magnetic resonance imaging data, we describe landmarks to help identify the position and borders of ' $h V 4$ '. The data consist of anatomical images, visualized as cortical meshes to highlight the sulcal and gyral patterns, and functional data obtained from retinotopic mapping experiments, visualized as eccentricity and angle maps on the cortical surface.

Several features of the functional and anatomical data can be found across nearly all subjects and are helpful for identifying the location and extent of the hV4 map. The medial border of hV4 is shared with the posterior, ventral portion of $\mathrm{V} 3$, and is marked by a retinotopic representation of the upper vertical meridian. The anterior border of hV4 is shared with the VO-1 map, and falls on a retinotopic representation of the peripheral visual field, usually coincident with the posterior transverse collateral sulcus. The ventro-lateral edge of the map typically falls on the inferior occipital gyrus, where functional MRI artifacts often obscure the retinotopic data. Finally, we demonstrate the continuity of retinotopic parameters between hV4 and its neighbors; hV4 and V3v contain iso-eccentricity lines in register, whereas hV4 and VO-1 contain iso-polar angle lines in register.

Together, the multiple constraints allow for a consistent identification of the hV4 map across most human subjects.

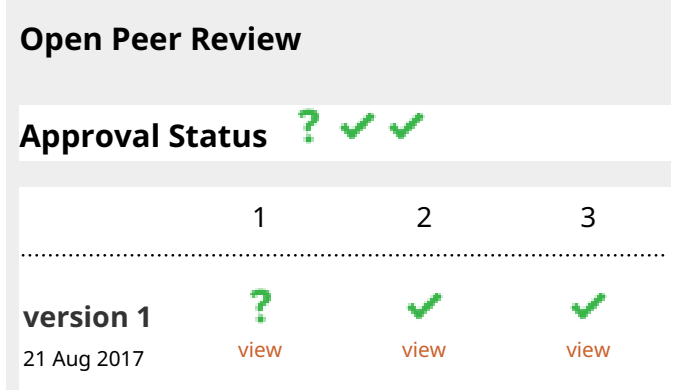

1. Ben M. Harvey, Utrecht University, Utrecht, The Netherlands

2. Geoffrey K. Aguirre (D), University of Pennsylvania, Philadelphia, USA

3. Antony B. Morland (iD), University of York, York, UK

Any reports and responses or comments on the article can be found at the end of the article. 


\section{Keywords}

visual cortex, functional magnetic resonance imaging, retinotopic map, visual field map, HV4, population receptive field, collateral

sulcus, fusiform gyrus

\section{‘ịncf}

This article is included in the INCF gateway.

Corresponding author: Jonathan Winawer (jonathan.winawer@nyu.edu)

Author roles: Winawer J: Conceptualization, Data Curation, Formal Analysis, Funding Acquisition, Investigation, Methodology, Project Administration, Resources, Software, Validation, Visualization, Writing - Original Draft Preparation, Writing - Review \& Editing; Witthoft N: Conceptualization, Data Curation, Formal Analysis, Investigation, Methodology, Project Administration, Resources, Software, Validation, Visualization, Writing - Original Draft Preparation, Writing - Review \& Editing

Competing interests: No competing interests were disclosed.

Grant information: The authors acknowledge two funding sources, National Institutes of Health (grant R00-EY022116 to JW) and (grant R01-EY023915 to Kalanit Grill Spector (supporting NW)).

The funders had no role in study design, data collection and analysis, decision to publish, or preparation of the manuscript.

Copyright: (c) 2017 Winawer J and Witthoft N. This is an open access article distributed under the terms of the Creative Commons Attribution License, which permits unrestricted use, distribution, and reproduction in any medium, provided the original work is properly cited.

How to cite this article: Winawer J and Witthoft N. Identification of the ventral occipital visual field maps in the human brain [version 1; peer review: 2 approved, 1 approved with reservations] F1000Research 2017, 6:1526

https://doi.org/10.12688/f1000research.12364.1

First published: 21 Aug 2017, 6:1526 https://doi.org/10.12688/f1000research.12364.1 


\section{Introduction}

The human brain contains well over a dozen visual field maps ${ }^{1-3}$. Identification of these maps has been a major success in the history of visual neuroscience. Because researchers can identify the same brain region across multiple measurements and diverse populations, scientific findings can be aggregated across studies to arrive at a better understanding of human brain function. The success of such aggregation, however, is limited by the accuracy with which a given region can be identified across individuals. There is little to no doubt about the position and borders of several maps. Primary visual cortex (V1) always lies on the Calcarine sulcus ${ }^{4-7}$, and its borders can be identified based on data from a single functional magnetic resonance imaging (fMRI) scanning session with high precision $^{8,9}$. V2 and V3 can also be identified quite accurately and routinely, and in fact the retinotopy parameters of all three maps can be reasonably estimated from the anatomy alone ${ }^{10,11}$.

In contrast, the fourth visual field map has proven more difficult to characterize ${ }^{12}$, with considerably less consistency in map definition across laboratories, compared to V1-V $3^{13-21}$. There are several reasons. Compared to V1-V3, the fMRI signals in hV4 are less reliably driven by simple contrast patterns ${ }^{22}$, the homology with animal models is less certain ${ }^{23}$, imaging artifacts can affect some parts of the map in many subjects ${ }^{15}$, and anatomical landmarks are less frequently used in map delineation. Recent work, however, has examined the hV4 map in some detail ${ }^{15,20}$, suggesting that one can identify hV4 and its neighbors (Figure 1) with reasonably good consistency across individuals.

In our view, successful map identification of the hV4 maps is aided by combining several sources of data. First, we consider the anatomy, especially the pattern of sulci and gyri. Second we use the angle map and eccentricity map derived from retinotopy measurements. Third, we use a metric of signal quality such as mean BOLD signal (or signal to noise ratio, or the quality of the retinotopic fit) to identify possible imperfections in the measurements that should

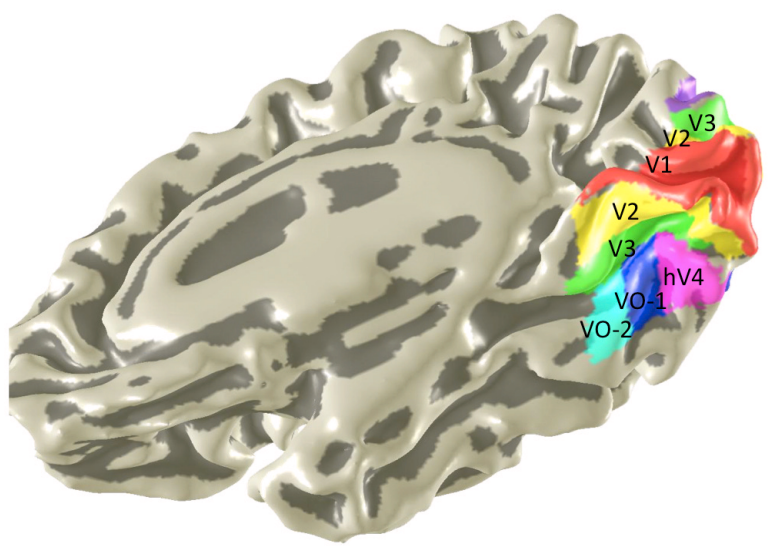

Figure 1. Location of ventral occipital visual field maps. The locations of 6 medial and ventral visual field maps are shown on a rendering of a subject's right hemisphere. The mesh underlay is a slightly smoothed rendering of the cortical surface. Sulci are indicated by dark gray and gyri by light gray. Adapted from 2. be ignored in identifying the map. In the simplest scenarios, all constraints agree and the map is easily identifiable. In some cases, the measurements do not fully agree but we can nonetheless identify the general location of $\mathrm{hV} 4$ and at least one or more of the map boundaries based on the mutual constraints.

The protocol for identifying the $\mathrm{hV} 4$ retinotopic map is described in the following text and in a step-by-step video description (Supplementary Movie 1).

\section{Protocol}

Our procedure for identifying visual field maps involves two kinds of MRI data and several processing steps. The required data are an anatomical image of the subject's brain (structural MRI) and functional data from a retinotopic mapping experiment. Typically, all data can be collected in less than one hour of scan time on a 3 Tesla scanner using a standard gradient echo pulse sequence for functional data and T1-weighted images for anatomical data. The analysis steps include segmenting gray and white matter to generate a cortical mesh, identifying the sulcal and gyral patterns in ventral occipito-temporal cortex, extracting the eccentricity and angle data from the retinotopic experiments, and then using these measurements to guide the marking of visual field boundaries.

There is considerable variability in the specific procedures used by different research groups for acquisition of functional and anatomical MRI data and for the derivation of retinotopy parameters from the fMRI scans. Any of the retinotopy methods in common usage is suitable; the only requirements are that one can visualize eccentricity and angle data on a cortical mesh. The methods to produce the images shown in this paper are described in more detail elsewhere ${ }^{20}$. We summarize these methods briefly and then focus on the specific steps for identifying the hV4 map and its neighbors.

\section{Data acquisition}

1.1. Anatomical data. Anatomical data are needed in order to render retinotopic data on surface representations. For subjects 1-3 depicted in the video presentation, 2-4 whole brain SPGR T1weighted MRI images were acquired on a $3 \mathrm{~T} \mathrm{GE} \mathrm{scanner} \mathrm{at} 1 \mathrm{~mm}$ isotopic resolution using an 8-channel whole-brain coil. The multiple images for each subject were aligned and averaged. Averaging multiple anatomical images is desirable as it increases the contrast of the boundary between the grey and white matter, and therefore aids segmentation and the creation of an accurate cortical surface.

1.2. Functional data. Functional data were acquired as $\mathrm{T} 2$ * $_{\text {- }}$ weighted gradient echo images with either a spiral ${ }^{24}$ or rectilinear (EPI) trajectory through k-space, with $2.5 \mathrm{~mm}$ isotropic voxels and a TR of either $1.5 \mathrm{~s}$ or $2 \mathrm{~s}$. (For details see ref 20.)

\section{Stimuli}

Retinotopy was measured with stimuli designed either for travelling wave analysis ${ }^{25}$ or population receptive field modeling ${ }^{26,27}$. For traveling wave analysis, alternate scans contained contrast patterns viewed through expanding ring apertures or clockwise rotating wedges with a visual field extent of 14 degrees from fixation. (For details see ref 20.) For population receptive field modeling, stimuli were contrast patterns viewed though moving bar apertures that 
slowly swept across the visual field, with a maximum eccentricity of 6 degrees from fixation. (For details see refs 15,26.)

\section{Functional MRI analysis}

3.1. Preprocessing. Several standard fMRI preprocessing steps were used prior to retinotopic analysis, including slice timing correction, motion correction, and high pass temporal filtering with a $1 / 20 \mathrm{~Hz}$ cutoff.

3.2. Travelling wave analysis. Data from traveling wave scans (wedges and rings) were analyzed voxel by voxel by applying the Fourier transform to the time series. The phase at the stimulus frequency measures the polar angle or eccentricity of the stimulus that most effectively drives the BOLD response in that voxel. The coherence of the signal at the stimulus frequency indicates the goodness of fit of the response to the periodic design. Coherence is defined as the power at the stimulus frequency divided by the power across all frequencies. (For details see ref 28.)

3.3. Population receptive fields. A population Receptive Field (pRF) model was solved for each voxel using methods described previously ${ }^{26}$. In brief, each pRF was defined as a 2-D isotropic Gaussian, parameterized by its center ( $\mathrm{x}, \mathrm{y}$ ), size (sd of Gaussian), and amplitude (scale factor). The model prediction is the dot product of the $\mathrm{pRF}$ and the stimulus aperture, convolved with a hemodynamic response function. The model was fit through a minimization of least squared error between the predicted and observed time series using a coarse-to-fine approach. The pRF center can be converted to polar coordinates to yield an angle and eccentricity map, similar to that obtained from traveling wave analysis. For the purposes of retinotopic mapping in this paper, other pRF parameters were not used (size and amplitude).

\section{Segmentation and visualization}

Because the retinotopic maps in visual cortex are organized on the 2D surface, not the 3D volume, map data is best visualized on a surface representation, typically rendered as an inflated (smoothed) or un-inflated 3D surface mesh, or as a flattened (2D) surface s9-31. $^{29}$. The data used for the surface rendering is usually the border of the gray and white matter, derived from a segmentation procedure that labels anatomical voxels from the T1 image as gray or white matter, and then finds the boundary that separates the two tissues. For the data shown in this paper, we segment the gray and white matter using the Freesurfer auto-segmentation tools ${ }^{32}$ followed by manual correction using ITK-GRAY software, modified from ITK-SNAP ${ }^{33}$. Functional data were then aligned to the whole brain anatomical data and rendered on a smoothed surface using custom software (vistasoft; http://vistalab.stanford.edu/software/).

\section{Anatomical landmarks}

We find it helpful to identify several sulci and gyri before drawing the retinotopic maps, and follow the naming conventions used by Duvernoy $^{34}$. The sulci and gyri labeled in Figure 2 can all be seen in post-mortem brains in Duvernoy's text (e.g., his Figure 17). Their locations are described briefly below.

5.1. Calcarine sulcus. The most useful landmark is the calcarine sulcus (sometimes called the calcarine fissure), which locates primary visual cortex (V1). It is the large sulcus in medial occipital

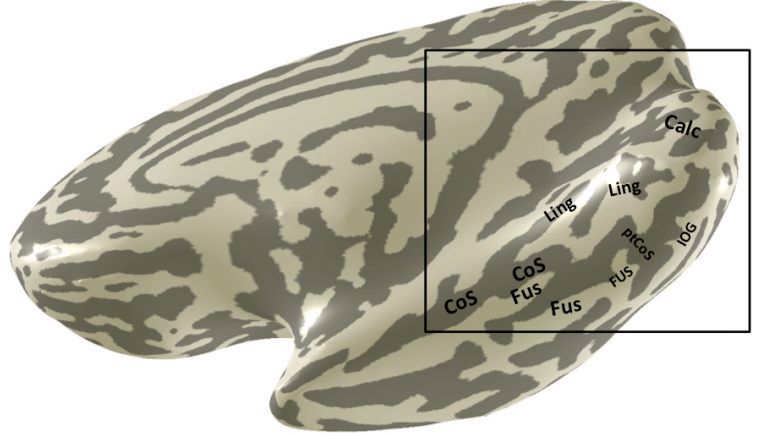

Figure 2. Anatomical landmarks in ventral occipitotemporal cortex. Several sulci and gyri are labeled on the ventral and medial surface of a highly smoothed right hemisphere. These sulci and gyri provide useful landmarks in the identification of the visual field maps. Calc: Calcarine sulcus; Ling: Lingual sulcus/gyrus; CoS: Collateral sulcus; Fus: Fusiform gyrus; ptCoS: Posterior transverse collateral sulcus; IOG: Inferior occipital gyrus.

cortex separating the lingual gyrus from the cuneus. It can be found unambiguously in every subject and is always the location of V1. It runs on an anterior-posterior axis, with the anterior end marked by the parietal occipital fissure, and the posterior end approximately at the occipital pole, though there is variability in the posterior extent, with the sulcus wrapping around to the lateral surface in some subjects, and terminating on the inferior surface in others ${ }^{35,36}$.

5.2. Lingual gyrus and lingual sulcus. The lingual gyrus is inferior to the calcarine sulcus and is the location of the ventral portions of V2 and V3. Its inferior boundary is the collateral sulcus. It stretches approximately from the posterior pole to the parahippocampal gyrus. The lingual gyrus contains one or more sulci together, considered the lingual sulcus.

5.3. Collateral sulcus. The collateral sulcus separates the lingual and parahippocampal gyri on the one side from the fusiform gyrus on the other. Its posterior end is usually denoted by a transverse portion of the sulcus, called the posterior transverse collateral sulcus (ptCoS). The ptCoS is an important landmark because it usually marks the division between the hV4 map and the VO- 1 map $^{20}$.

5.4. Fusiform gyrus. The fusiform gyrus is inferior to the lingual and parahippocampal gyrus, and is bounded medially by the collateral sulcus. The foveal representation of the ventral-occipital maps (VO-1/2) lies in the posterior portion of the fusiform gyrus.

5.5. Inferior occipital gyrus. The inferior occipital gyrus lies posterior to the fusiform gyrus and collateral sulcus and inferior to the lingual gyrus. It is generally separated from the posterior portion of the posterior fusiform gyrus by the posterior transverse collateral sulcus. The lateral portion of the hV4 map often lies here. This gyrus is typically in close proximity to the transverse venous sinus, which can lead to fMRI artifacts that obscure this portion of the hV4 map $^{15}$.

\section{Drawing the maps}

6.1. V1-V3 maps. Identification of the first three visual field maps in humans was one of the first accomplishments in visual 
neuroscience following the advent of $\mathrm{fMRI}^{25,28,37}$. Delineation of these map boundaries is now routine, although tracing the maps through the foveal confluence can be difficult in the absence of methods developed specifically for this purpose ${ }^{10}$. In brief, the V1 map is centered on the calcarine sulcus, and its borders with V2 lie on retinotopic representations of the vertical meridian. The peripheral boundary is usually not identified with fMRI because the field of view that can be achieved during scanning is less than the field of view represented in the maps. Hence the peripheral boundary of a region of interest is usually chosen as the most anterior region of the maps where the signal is good, according to a threshold in coherence (traveling wave analysis) or variance explained (pRF analysis). If there are clear reversals in the angle map all the way back to the occipital pole, then it may be possible to trace $\mathrm{V} 2$ and $\mathrm{V} 3$ in concentric ' $\mathrm{V}$ ' shapes around V1; otherwise the most foveal portion of the maps is typically labeled as a non-specific 'foveal confluence', and each map is traced as far into the fovea as the resolution in the angle maps allow, usually one or two degrees from fixation, such that the two arms of the ' $\mathrm{V}$ ' are not connected, and labeled as the dorsal and ventral portions of the map The dorsal and ventral V2/V3 borders are marked by a representation of the horizontal meridian in the angle map.

6.2. The hV4 map. The hV4 map lies on the ventral surface of the occipital lobe and borders at least two other visual field maps, the ventral part of V3 (V3v) and VO-1.

6.2.1. The anterior boundary: $h V 4 / V O-1$ The boundary between $\mathrm{hV} 4$ and VO-1 can be identified by both anatomical and retinotopic data. The retinotopic feature that defines this border is a reversal in the eccentricity map (Figure 3). This peripheral eccentricity band dividing hV4 from VO-1 usually coincides with a particular anatomical feature, the $\mathrm{ptCoS}^{20}$.
6.2.2. The medial boundary: $h V 4 / V 3 v$ One of the borders of $\mathrm{hV} 4$ is shared with the ventral portion of V3 (V3v). This border is defined by an upper vertical meridian polar angle reversal (Figure 4). Because the V1-V3 maps are typically well defined by the retinotopic data, it is usually also clear where this boundary is found.

6.2.3. The ventral/lateral boundary: The ventral/lateral boundary of hV4 is more difficult to identify than the other boundaries because there is no unambiguous feature of the retinotopic data to define it. As described in 6.2.1 and 6.2.2, we know what is on the other side of two of the hV4 boundaries, making those boundaries well defined: The anterior boundary is defined by an eccentricity reversal and is shared with VO-1, and the medial boundary is defined by a polar angle reversal and is shared with $\mathrm{V} 3 \mathrm{v}$. In contrast, there is not a well-established map or retinotopic feature abutting the ventral side of hV4. Furthermore, there is often signal dropout in the fMRI measure on the ventral / lateral aspect of the hV4 map due to the transverse venous sinus, which typically runs near the inferior occipital gyrus ${ }^{15}$. In some cases, the signal dropout defines the most ventral/lateral extent of the map that can be identified (Figure 5 and Figure 6).

6.2.4. Iso-eccentricity lines shared by hV4 and V3v: The general organization of the hV4 map and its neighbors can be understood by tracing iso-eccentricity and iso-angle lines. The hV4 eccentricity map is in register with the V1-V3 maps, so that iso-eccentricity lines span the hV4/V3v border (Figure 7). Unlike the V1-V3 maps, the hV4 map appears to contain little representation of the far periphery; this may be because the neurons in $\mathrm{hV} 4$ do not respond to stimuli in the periphery, or it may be because peripheral representation is highly compressed into a small amount of cortex, such that responses to more foveal stimuli have a much larger effect on the

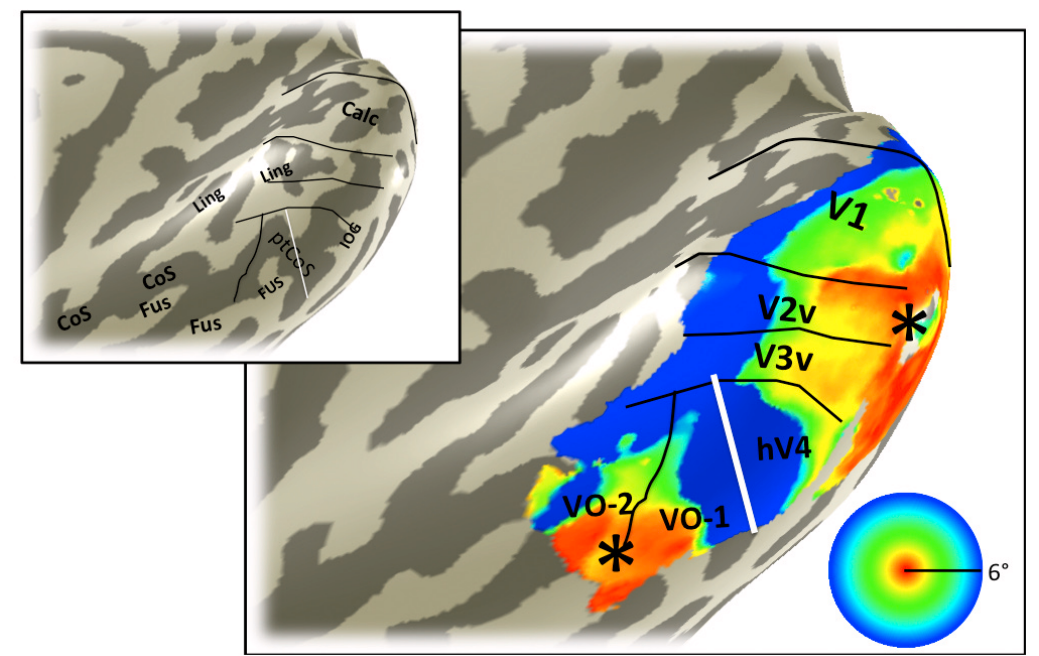

Figure 3. Eccentricity map. The color overlay on the smoothed cortical mesh in the main panel shows the stimulus eccentricity that most effectively drives each cortical location. The eccentricity was derived from solving a pRF model, and the stimulus extent was limited to 6 degrees. The asterisk indicates the confluent foveal representation. Black and white lines mark the boundaries between visual field maps. The white line divides hV4 from VO-1 and is the only boundary line in this figure that is derivable from the eccentricity map. This line coincides with the ptCoS (inset) and with the eccentricity reversal (blue in color overlay). Hence the white line also divides the ventral occipital maps into two clusters, one that includes V1-hV4, and one that includes VO-1/2 ${ }^{17}$. Data are limited to voxels in which the pRF prediction accounted for at least $10 \%$ of the variance explained in the time series and to a posterior mask that includes the 6 labeled visual field maps: V1, V2 and V3 ventral, hV4, VO-1, and VO-2. The inset is the same cortical mesh with labels indicating the major sulcal and gyral patterns, as in Figure 2. 


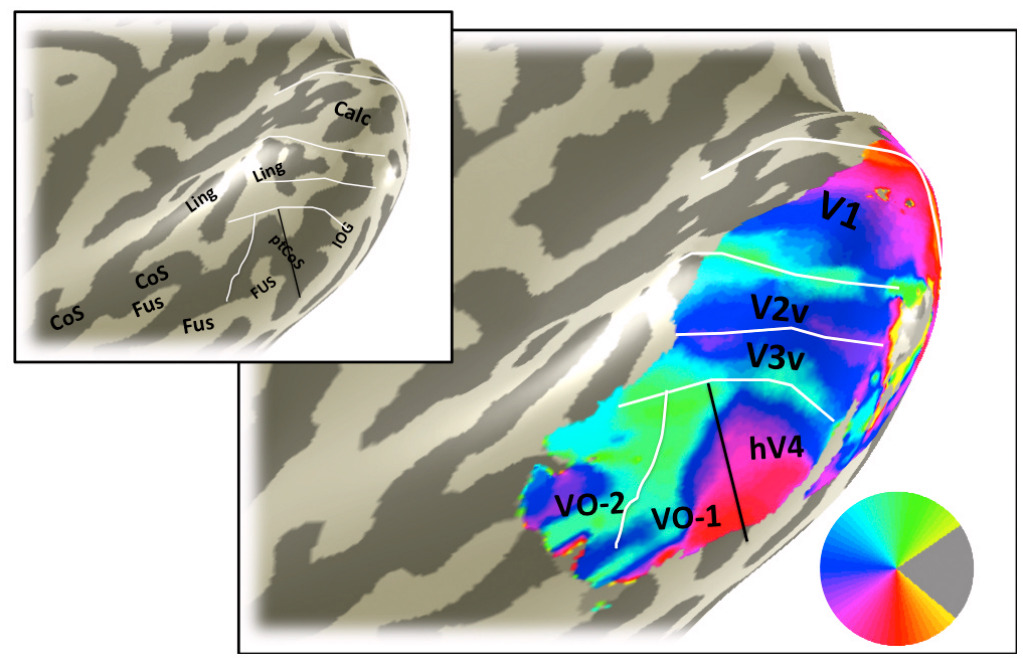

Figure 4. Angle map. The color overlay in the main panel indicates the angle in the visual field that most effectively drives responses in each cortical location. The visual field map boundaries in white can be identified from the angle map. Otherwise as Figure 3.

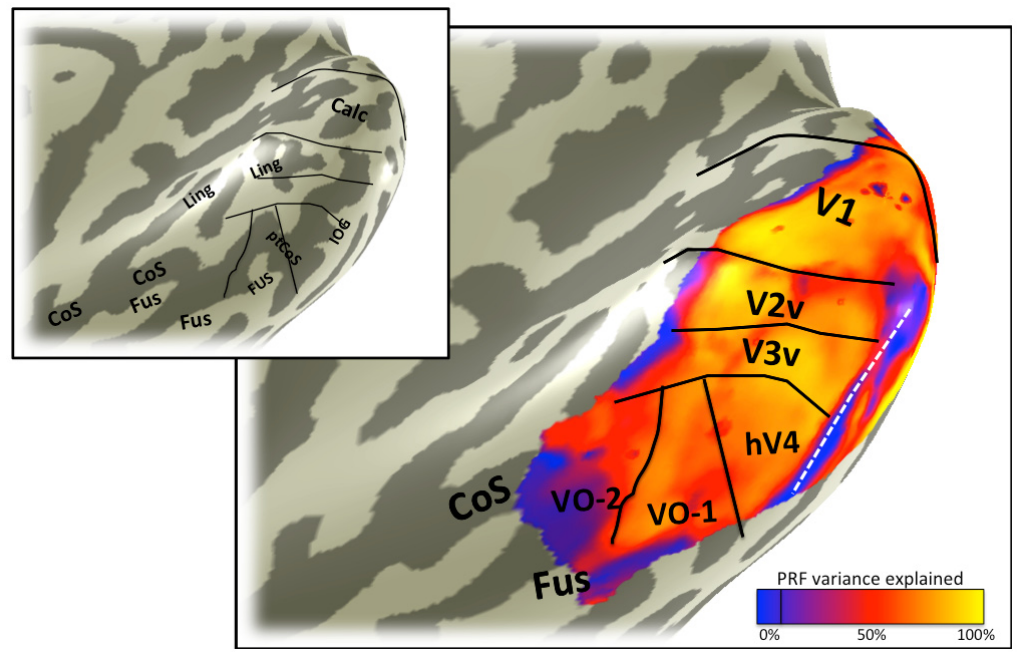

Figure 5. Variance explained map. The color overlay in the main panel indicates variance in the voxel time series explained by the pRF model predictions. Otherwise as Figure 3. Some locations with low variance explained are likely due to fMRI artifacts, such as the region indicated by the white line, where the low variance explained is caused by dropout from the transverse sinus. Other regions with low variance explained may have visual field representations outside the stimulus extent, such as the anterior edge of V1, V2, and V3. 


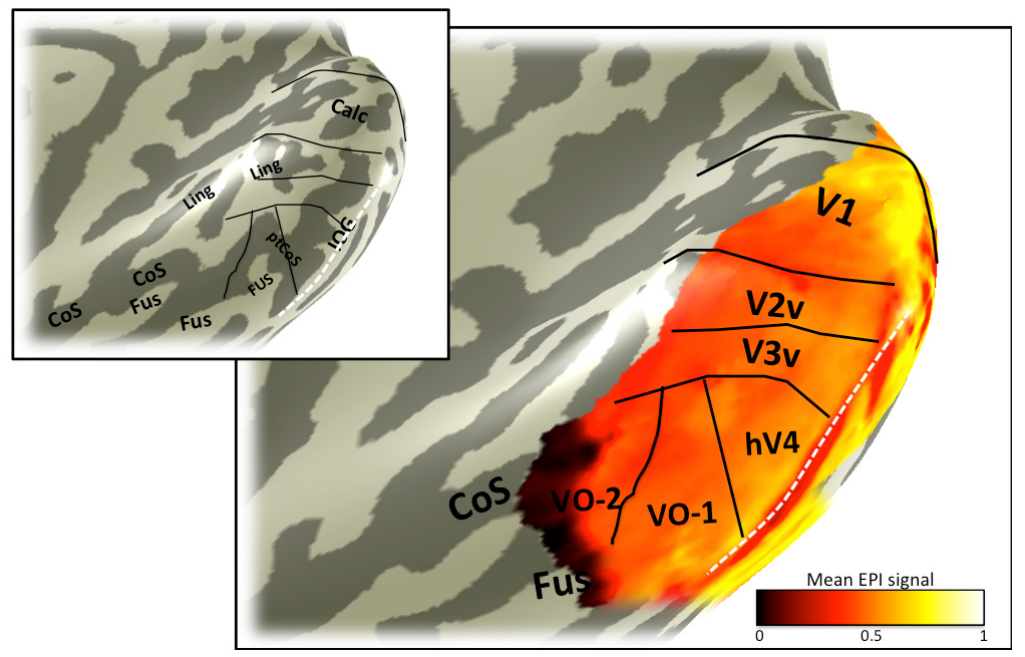

Figure 6. Mean signal map. The color overlay in the main panel indicates the mean fMRI signal. The signal is not uniform across cortex. Certain regions have low mean signal due scanning artifacts. The region indicated by the white line lies near the transverse venous sinus which causes signal dropout. Retinotopic data from these locations must be interpreted with caution. Otherwise as Figure 3.

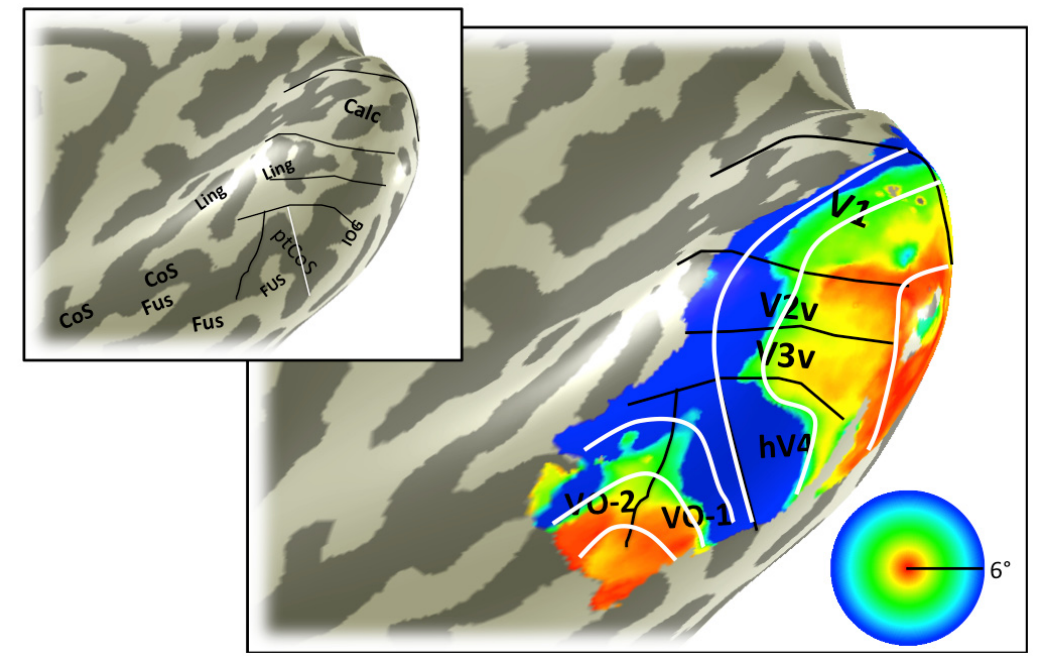

Figure 7. Iso-eccentricity lines. Iso-eccentricity lines in visual cortex are continuous within clusters, such as the posterior cluster containing $\mathrm{V} 1, \mathrm{~V} 2, \mathrm{~V} 3$, and $\mathrm{hV} 4$, and the ventral occipital cluster containing the VO-1/2 maps. The eccentricity overlay and the map boundaries are identical to those in Figure 3.

BOLD time course, masking the peripheral representation. Along the posterior-to-anterior axis, hV4 is therefore much shorter than $\mathrm{V} 1-\mathrm{V} 3$, and the most anterior eccentricity band in hV4 crossed V3v near the middle of the length of $\mathrm{V} 3 \mathrm{v}$.

6.2.5. Iso-angle lines shared by $\mathbf{h V 4}$ and VO-1: The iso-angle lines in $\mathrm{hV} 4$ are continuous with the VO-1 maps and not the V3v map. The lines usually bend along the hV4/V0-1 border, with the lower meridian representation being the most ventral and the shortest isoangle line (Figure 8).

\section{Results}

The sulci and gyri associated on the ventral occipital cortical surface from a representative subject (S1) are shown in Figure 2. The most useful anatomical landmarks for locating the visual field maps are the calcarine sulcus (V1); the lingual gyrus and lingual sulcus $(\mathrm{V} 2 \mathrm{v} / \mathrm{V} 3 \mathrm{v})$; the posterior collateral sulcus and inferior occipital gyrus, which bound hV4; and the fusiform gyrus and collateral sulcus, where the VO maps are found.

The eccentricity map for subject S1 shows the large-scale organization of the maps (Figure 3). The key feature on the 


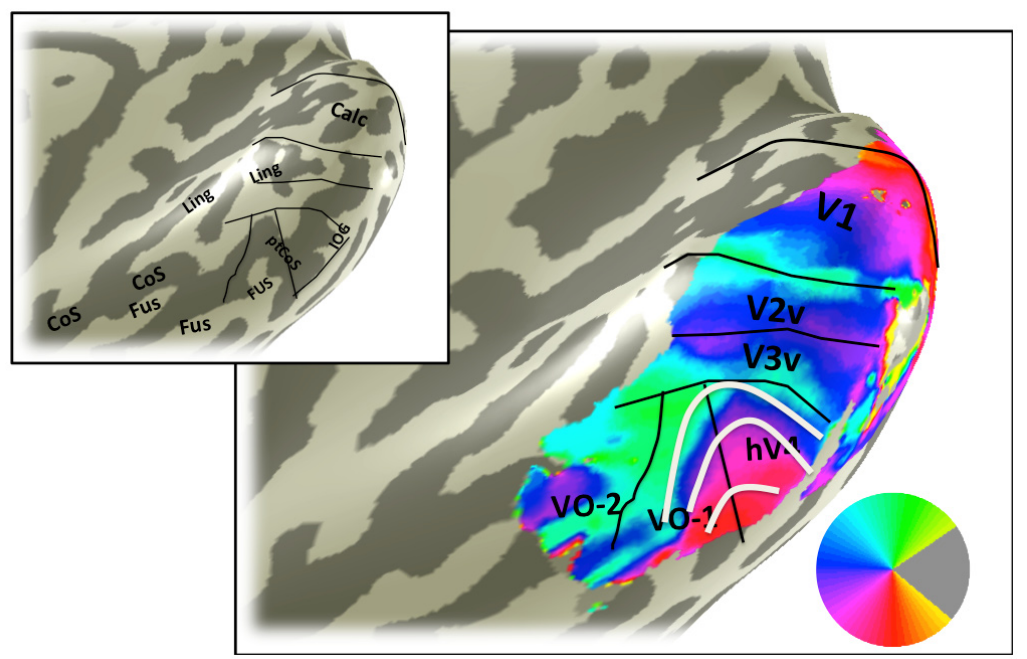

Figure 8. Iso-angle lines. The iso-angle lines in hV4 and VO-1, indicated by white lines, are continuous across the two map clusters. Otherwise as Figure 4.

eccentricity map for identifying hV4 is the peripheral representation within the ptCoS, which marks the hV4/VO-1 boundary. The angle map (Figure 4) is used to define the V3v/hV4 boundary as well as the VO-1/VO-2 boundary.

FMRI signal quality is not uniform across the cortical surface. Some locations have poor signal due to known measurement artifacts, such as those which arise in regions near large sinuses. In subject $\mathrm{S} 1$, the transverse venous sinus corrupts the fMRI signal on the inferior occipital gyrus, resulting in low variance explained by the pRF model (Figure 5) and signal dropout (Figure 6) in this region.

Iso-eccentricity and iso-angle lines in subject $\mathrm{S} 1$ clarify the internal structure of the hV4 map and the relationship between hV4 and its neighbors. The iso-eccentricity lines are in register across $\mathrm{hV} 4$ and V1-V3 (Figure 7). The iso-angle lines are continuous across the hV4/VO-1 boundary (Figure 8).

Angle maps and eccentricity maps from additional subjects show similar patterns to S1. The V1-V3, hV4 and VO-1/2 maps are shown for a representative right hemisphere (S2; Figure 9; Figure 10) and left hemisphere (S3; Figure 11; Figure 12). The large scale organization is similar for all subjects. For example, there is an eccentricity reversal dividing $\mathrm{hV} 4$ and $\mathrm{VO}-1$ and an angle reversal dividing $\mathrm{hV} 4$ and $\mathrm{V} 3$. In all subjects, the eccentricity reversal falls on or near the ptCoS. Some details differ between subjects. For example, the foveal representation in hV4 is clear in S1 and S2 but not S3 (Figure 11), likely due to corrupted signal from draining veins. A second difference across subjects is that the upper meridian angle reversal dividing VO-1 and VO-2 is clear in S1 and S3 but not S2 (Figure 10). Nonetheless, there is sufficient regularity across subjects to identify the principal features defining the hV4 map and its neighbors, V3v and VO-1 (Figure 13).

\section{Discussion}

Regularity of maps and anatomy

Identifying visual field maps is an important component of characterizing the organization and function of visual cortex. It is important to have well-justified and reproducible methods to define the maps. In the case of the V1-V3 maps, the functional and anatomical organization is sufficiently regular and well understood that these maps can be identified using automated procedures (no human intervention $)^{11,12}$. The hV4 and VO maps are not yet included in automated fitting procedures; however, recent progress suggests that these maps too have a high degree of regularity. Two boundaries are well defined by retinotopic features (sections 6.2.2, 6.2.3), and one of these also coincides with an anatomical landmark, the ptCoS. Moreover, the internal structure of the hV4 map and its neighbors are well understood, such that the iso-eccentricity and iso-angle lines derived from retinotopic mapping follow regular patterns.

\section{Variability across subjects}

While most of the large-scale structures in the ventral occipital retinotopic maps are similar across subjects, there are also individual differences. Some of these differences likely reflect quantitative differences between subjects in the size and layout of the maps. 


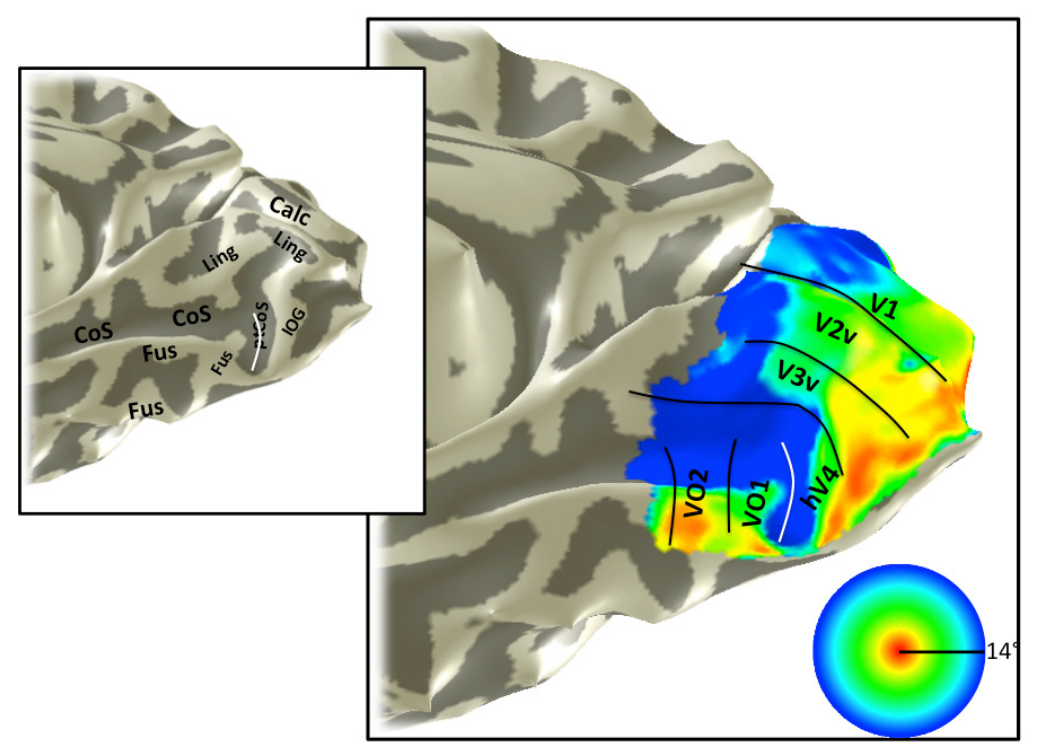

Figure 9. Eccentricity map, S2. An eccentricity map is shown on the partially inflated cortical surface of subject 2's right hemisphere. The stimulus extent was 14 degrees and the data are from traveling wave analysis of expanding rings. Otherwise as Figure 3.

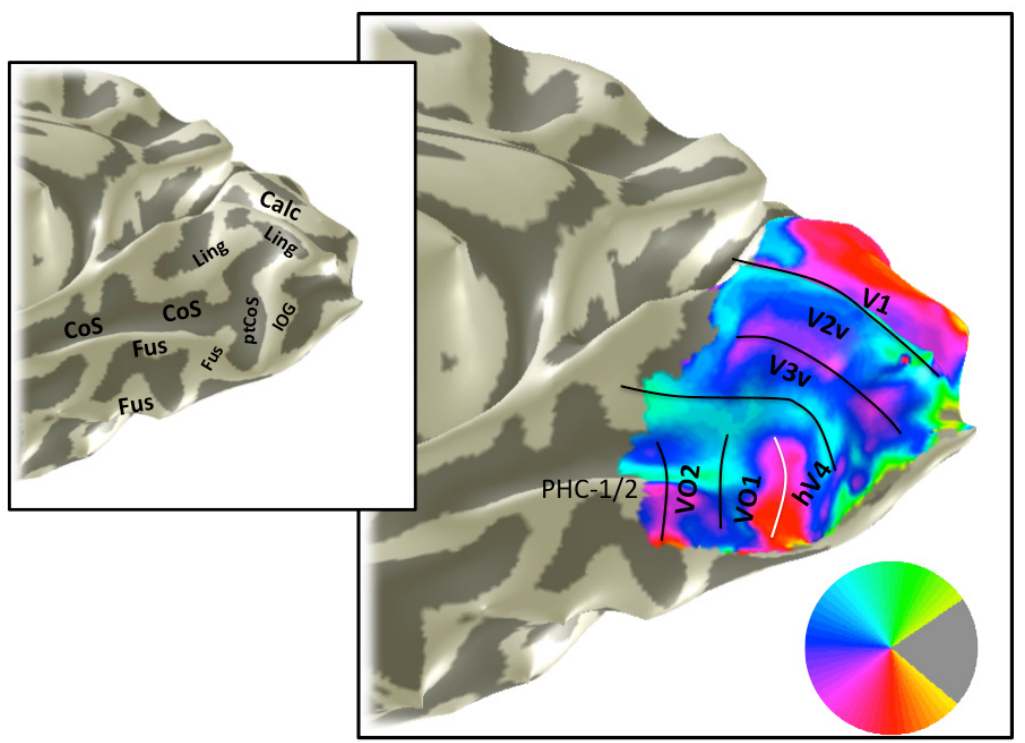

Figure 10. Angle map, S2. An angle map is shown for subject 2's right hemisphere. Otherwise as Figure 9 and Figure 3. 


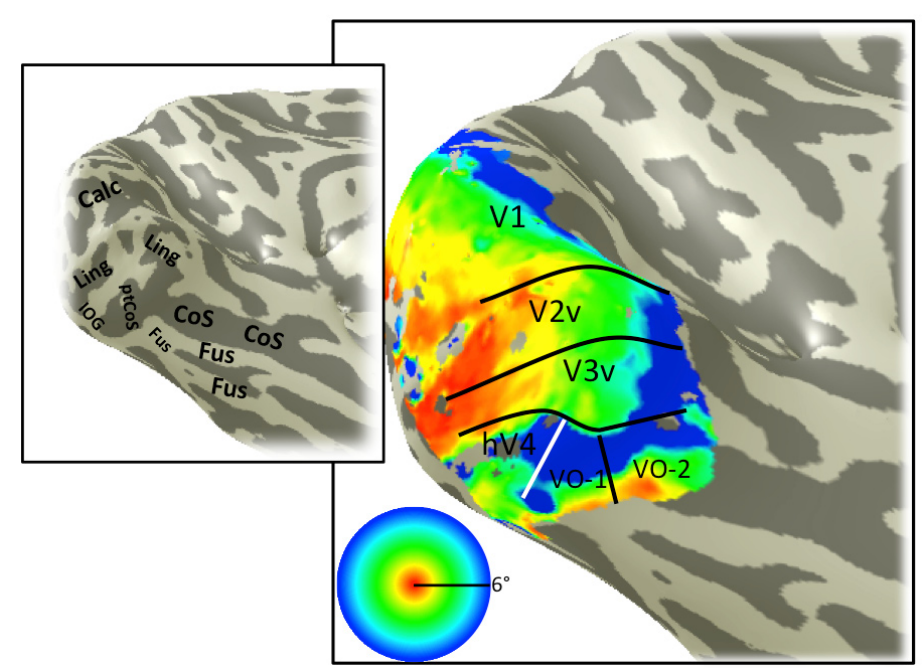

Figure 11. Eccentricity map, S3. An example of a left hemisphere eccentricity map derived from pRF model fitting. Otherwise as Figure 3.

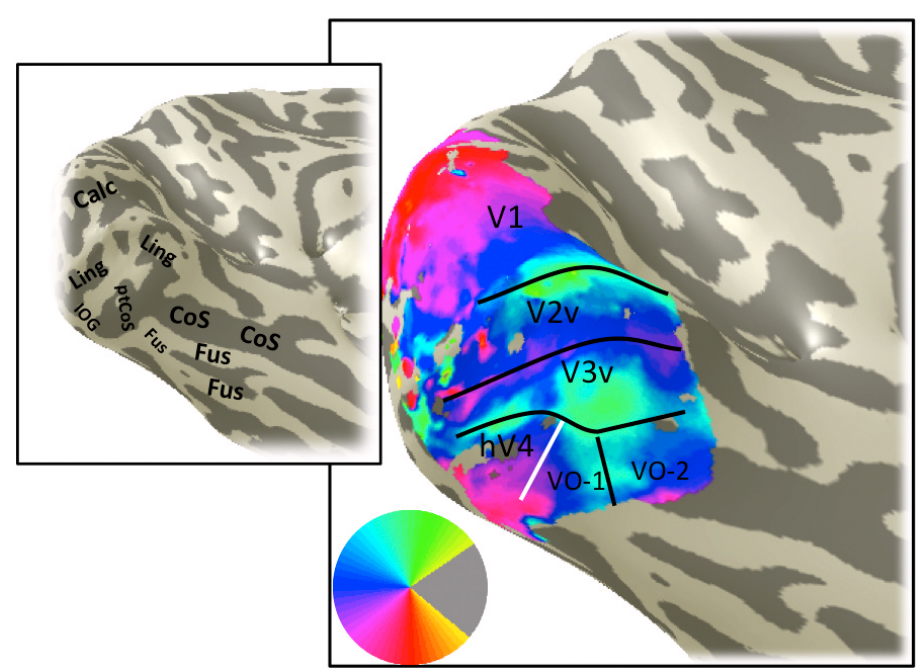

Figure 12. Angle map, S3. An example of a left hemisphere angle map derived from pRF model fitting. Otherwise as Figure 4. 


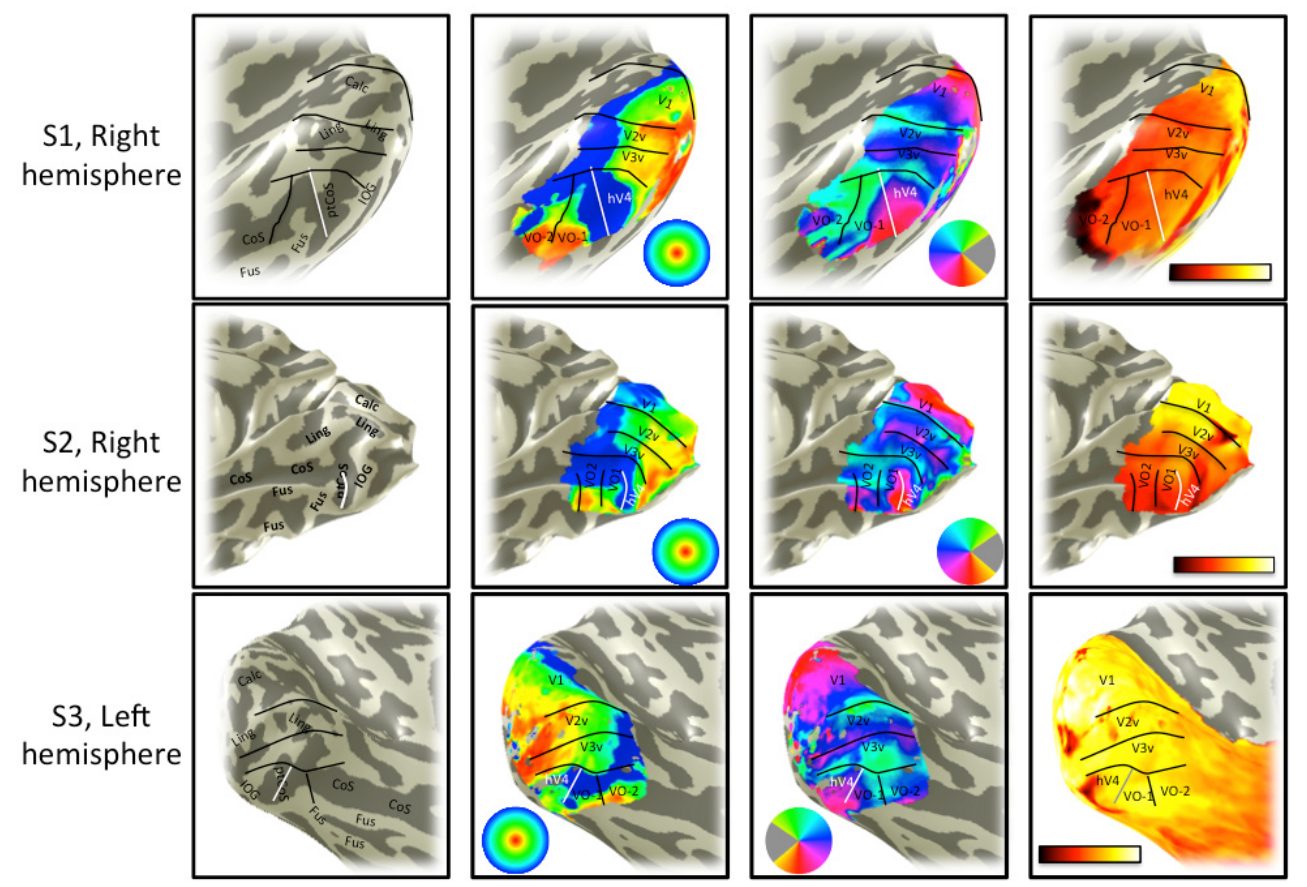

Figure 13. Map comparison, S1-S3. Four types of data are shown for three subjects: sulcal and gyral landmarks (column 1), eccentricity (column 2), angle (column 3), and mean fMRI signal (column 4). Comparisons of the datasets reveal regularities across subjects, such as the fact that the ptCoS is well aligned with the hV4/VO-1 boundary defined by an eccentricity reversal (white line in columns 1 and 2). There are also differences across subjects. For example the foveal representation of hV4 is less clear in S3 than in S1 and S2.

Other differences reflect various sources of measurement noise. In no case will a measured map exactly match a template, and in some cases map boundaries will be ambiguous. We believe that the best approach offered is to simultaneously satisfy as many of the constraints from the anatomy, eccentricity, and angle maps as possible.

\section{Data availability}

The functional and anatomical data for subjects 1-3 have been de-identified and made publicly available on the Open Science Framework (http://doi.org/10.17605/OSF.IO/UYHMX ${ }^{38}$ ). Analysis code written in Matlab (Mathworks; Natick, MA) is also available via the same site. The analysis code reproduces the images of the cortical meshes with color overlays as shown in Figure 3-Figure 13.

\section{Competing interests}

No competing interests were disclosed.

\section{Grant information}

The authors acknowledge two funding sources, National Institutes of Health (grant R00-EY022116 to JW) and (grant R01-EY023915 to Kalanit Grill Spector (supporting NW)).

The funders had no role in study design, data collection and analysis, decision to publish, or preparation of the manuscript.

\section{Supplementary Material}

Supplementary Movie 1: Method for identifying the ventral occipital retinotopic maps.

Click here to access the data. 
1. Wandell BA, Winawer J: Imaging retinotopic maps in the human brain. Vision Res. 2011; 51(7): 718-37. PubMed Abstract | Publisher Full Text | Free Full Text

2. Wandell BA, Dumoulin SO, Brewer AA: Visual field maps in human cortex. Neuron. 2007; 56(2): 366-83. PubMed Abstract | Publisher Full Text

3. Wang L, Mruczek RE, Arcaro MJ, et al:: Probabilistic Maps of Visual Topography in Human Cortex. Cereb Cortex. 2015; 25(10): 3911-31. PubMed Abstract | Publisher Full Text | Free Full Text

4. Henschen SE: On the visual path and centre. Brain. 1893; 16(1-2): 170-80 Publisher Full Text

5. Inouye T: Die Sehstörungen bei Schussverletzungen der kortikalen Sehsphäre: nach Beobachtungen an Verwundeten der letzten japanischen Kriege. W. Engelmann; 1909

Reference Source

6. Holmes G: Disturbances of Vision by Cerebral Lesions. Br J Ophthalmol. 1918; 2(7): 353-84

PubMed Abstract | Publisher Full Text | Free Full Text

7. Horton JC, Hoyt WF: The representation of the visual field in human striate cortex. A revision of the classic Holmes map. Arch Ophthalmol. 1991; 109(6): $816-24$

PubMed Abstract | Publisher Full Text

8. Kirson D, Huk AC, Cormack LK: Quantifying spatial uncertainty of visual area boundaries in neuroimaging data. J Vis. 2008; 8(10): 101-5. PubMed Abstract | Publisher Full Text

9. Schira MM, Tyler CW, Breakspear M, et al:: The foveal confluence in human visual cortex. J Neurosci. 2009; 29(28): 9050-8.

PubMed Abstract | Publisher Full Text

10. Benson NC, Butt OH, Brainard DH, et al:: Correction of distortion in flattened representations of the cortical surface allows prediction of V1-V3 functiona organization from anatomy. PLoS Comput Biol. 2014; 10(3): e1003538. PubMed Abstract | Publisher Full Text | Free Full Text

11. Benson NC, Butt $\mathrm{OH}$, Datta R, et al:: The retinotopic organization of striate cortex is well predicted by surface topology. Curr Biol. 2012; 22(21): 2081-5. PubMed Abstract | Publisher Full Text | Free Full Text

12. Winawer J, Witthoft N: Human $\mathbf{V} 4$ and ventral occipital retinotopic maps. Vis Neurosci. 2015; 32: E020.

PubMed Abstract | Publisher Full Text | Free Full Text

13. Wade AR, Brewer AA, Rieger JW, et al:: Functional measurements of human ventral occipital cortex: retinotopy and colour. Philos Trans $R$ Soc Lond B Biol Sci. 2002; 357(1424): 963-73.

PubMed Abstract | Publisher Full Text | Free Full Text

14. Hansen KA, Kay KN, Gallant JL: Topographic organization in and near human visual area V4. J Neurosci. 2007; 27(44): 11896-911. PubMed Abstract | Publisher Full Text

15. Winawer J, Horiguchi H, Sayres RA, et al.: Mapping hV4 and ventral occipital cortex: the venous eclipse. J Vis. 2010; 10(5): 1. PubMed Abstract | Publisher Full Text | Free Full Text

16. Hadjikhani N, Liu AK, Dale AM, et al:: Retinotopy and color sensitivity in human visual cortical area V8. Nat Neurosci. 1998; 1(3): 235-41. PubMed Abstract | Publisher Full Text

17. Brewer AA, Liu J, Wade AR, et al.: Visual field maps and stimulus selectivity in human ventral occipital cortex. Nat Neurosci. 2005; 8(8): 1102-9. PubMed Abstract | Publisher Full Text

18. Tootell RB, Hadjikhani N: Where is 'dorsal V4' in human visual cortex? Retinotopic, topographic and functional evidence. Cereb Cortex. 2001; 11(4): 298-311.

PubMed Abstract | Publisher Full Text

19. Larsson J, Heeger DJ: Two retinotopic visual areas in human lateral occipital cortex. J Neurosci. 2006; 26(51): 13128-42. PubMed Abstract | Publisher Full Text | Free Full Text

20. Witthoft N, Nguyen ML, Golarai G, et al:: Where Is Human V4? Predicting the Location of hV4 and VO1 from Cortical Folding. Cereb Cortex. 2014; 24(9):
$2401-8$

PubMed Abstract | Publisher Full Text | Free Full Text

21. Zeki S, Watson JD, Lueck CJ, et al.: A direct demonstration of functional specialization in human visual cortex. J Neurosci. 1991; 11(3): 641-9. PubMed Abstract

22. Kay KN, Naselaris T, Prenger RJ, et al.: Identifying natural images from human brain activity. Nature. 2008; 452(7185): 352-5. PubMed Abstract | Publisher Full Text | Free Full Text

23. Meadows JC: Disturbed perception of colours associated with localized cerebral lesions. Brain. 1974; 97(4): 615-32.

PubMed Abstract | Publisher Full Text

24. Glover GH, Lai S: Self-navigated spiral fMRI: interleaved versus single-shot. Magn Reson Med. 1998; 39(3): 361-8. PubMed Abstract | Publisher Full Tex

25. Engel SA, Rumelhart DE, Wandell BA, et al.: fMRI of human visual cortex. Nature. 1994; 369(6481): 525

PubMed Abstract | Publisher Full Text

26. Dumoulin SO, Wandell BA: Population receptive field estimates in human visual cortex. Neuroimage. 2008; 39(2): 647-60.

PubMed Abstract | Publisher Full Text | Free Full Text

27. Wandell BA, Winawer J, Kay KN: Computational modeling of responses in human visual cortex. In: Toga A, editor. Brain Mapping: An Encyclopedic Reference. 2015; 1: 651-659.

Publisher Full Text

28. Engel SA, Glover GH, Wandell BA: Retinotopic organization in human visual cortex and the spatial precision of functional MRI. Cerebral Cortex. 1997; 7(2): 181-92.

PubMed Abstract | Publisher Full Text

29. Teo PC, Sapiro G, Wandell BA: Creating connected representations of cortica gray matter for functional MRI visualization. IEEE Trans Med Imaging. 1997 16(6): 852-63.

PubMed Abstract | Publisher Full Tex

30. Wandell BA, Chial S, Backus BT: Visualization and measurement of the cortical surface. J Cogn Neurosci. 2000; 12(5): 739-52. PubMed Abstract | Publisher Full Text

31. Carman GJ, Drury HA, Van Essen DC: Computational methods fo reconstructing and unfolding the cerebral cortex. Cereb Cortex. 1995; 5(6): 506-17.

PubMed Abstract | Publisher Full Text

32. Dale AM, Fischl B, Sereno MI: Cortical surface-based analysis. I. Segmentation and surface reconstruction. Neuroimage. 1999; 9(2): 179-94. PubMed Abstract | Publisher Full Text

33. Yushkevich PA, Piven J, Hazlett HC, et al:: User-guided 3D active contour segmentation of anatomical structures: Significantly improved efficiency and reliability. Neuroimage. 2006; 31(3): 1116-28. PubMed Abstract | Publisher Full Text

34. Duvernoy HM: The human brain: surface, three-dimensional sectional anatomy with MRI, and blood supply. 2nd completely rev. and enl. ed. Wien; New York: Springer; 1999; 491. Publisher Full Text

35. Dumoulin SO, Hoge RD, Baker CL Jr, et al.: Automatic volumetric segmentation of human visual retinotopic cortex. Neuroimage. 2003; 18(3): 576-87. PubMed Abstract | Publisher Full Text

36. Stensaas SS, Eddington DK, Dobelle WH: The topography and variability of the primary visual cortex in man. J Neurosurg. 1974; 40(6): 747-55. PubMed Abstract | Publisher Full Text

37. Sereno MI, Dale AM, Reppas JB, et al.: Borders of multiple visual areas in humans revealed by functional magnetic resonance imaging. Science. 1995; 268(5212): 889-93.

PubMed Abstract | Publisher Full Text

38. Winawer J, Witthoft N: Identification of the Ventral Occipital Visual Field Maps in the Human Brain. Open Science Framework. 2017.

Data Source 


\section{Open Peer Review}

\section{Current Peer Review Status:}

\section{Version 1}

Reviewer Report 06 November 2017

https://doi.org/10.5256/f1000research.13389.r27268

(c) 2017 Morland A. This is an open access peer review report distributed under the terms of the Creative Commons Attribution License, which permits unrestricted use, distribution, and reproduction in any medium, provided the original work is properly cited.

\section{Antony B. Morland}

Department of Psychology, York Neuroimaging Centre, University of York, York, UK

I rather like this short paper that reports on how to identify ventral visual field maps. Having had to attempt to do this on many occasions, sometimes with limited success, I find the paper very useful in giving evidence based guidance that could really help. The video and figures that support the paper are excellent and offer a resource for early career researchers to build up confidence in what can be a challenging task.

One suggestion I have that might broaden the appeal of the study is to reflect a bit more on the relationship between the layout found in human and that in macaque. V4 is split between dorsal and ventral representations of quadrants, whereas the human data are often consistent with the forth (ventral) map being a representation of a hemifield. The authors touch on this early on, but having presented their data, they may reflect further on this in the Discussion.

\section{Minor points and suggestions}

'The multiple images for each subject were aligned and averaged. Averaging multiple anatomical images is desirable as it increases the contrast of the boundary between the grey and white matter, and therefore aids segmentation and the creation of an accurate cortical surface.'

I agree, but the authors might also point out that multiple, relatively short, acquisitions are also probably better than using longer acquisitions during which participant motion could work against benefits in contrast.

I understood every word of the section that described the functional methods, but I would because I pretty much use the same methods. Are more details required for a naive reader? Anatomical Figure (Figure 2) I think it would be good to use colour to mark the sulci/gyri and colour the labelling text in line with the sulcal colour. That way repeats of lettering can be avoided and less ambiguity between the location of text and the anatomical feature can be achieved.

Section on calcarine sulcus. Perhaps a bit more text on the variants observed at the pole - there is a reasonably well-documented ' $Y$ ' configuration too, I think. 
Section 6.1 'The peripheral boundary is usually not identified with fMRI because the field of view that can be achieved during scanning is less than the field of view represented in the maps.' $I$ assume this refers the the size of the stimulus, not the FOV used in imaging, but some clarification is needed here.

'and each map is traced as far into the fovea as the resolution in the angle maps allow, usually one or two degrees from fixation'

I think representation needs to be inserted here a couple of times e.g. 'as far into the representation of the fovea' and 'usually at the representation of one or two degrees from fixation'

Is the rationale for developing the new method (or application) clearly explained? Yes

Is the description of the method technically sound?

Yes

Are sufficient details provided to allow replication of the method development and its use by others?

Yes

If any results are presented, are all the source data underlying the results available to ensure full reproducibility?

Yes

Are the conclusions about the method and its performance adequately supported by the findings presented in the article?

Yes

Competing Interests: No competing interests were disclosed.

Reviewer Expertise: fMRI, vision

I confirm that I have read this submission and believe that I have an appropriate level of expertise to confirm that it is of an acceptable scientific standard.

Reviewer Report 06 September 2017

https://doi.org/10.5256/f1000research.13389.r25204

(C) 2017 Aguirre G. This is an open access peer review report distributed under the terms of the Creative Commons Attribution License, which permits unrestricted use, distribution, and reproduction in any medium, provided the original work is properly cited. 


\section{Geoffrey K. Aguirre \\ Department of Neurology, University of Pennsylvania, Philadelphia, PA, USA}

Winawer and Witthoft describe a standard procedure to define human visual area hV4 within retinotopic mapping data obtained using fMRI. The definition of the early, retinotopically organized visual areas is a crucial first step in many studies of the cortical organization for vision. While areas V1-V3 are readily identified within functional MRI mapping data, area hV4 is often challenging due to reduced signal strength, imaging artifacts, and the peculiar organization of this region.

The paper documents and supports a supplementary video, which is primarily a presentation of the figures from the paper. This video, narrated in the even, velvet tones of the first author, serves as a very nice introduction to ventral retinotopic maps generally, and to the specific procedure of identifying the borders of area hV4. The video is a sufficiently important resource that I suggest that the authors mention it in the abstract.

I have no concerns regarding the method or the conclusions of the paper. Instead, I have two things that I would have ideally liked to have seen discussed:

First, for the purposes of this report, I think it is appropriate for the authors to assume that the reader has in hand a retinotopic map that is displayed on an inflated cortical surface. They are therefore justified in providing minimal treatment of the stimulus, MRI acquisition parameters, and initial analysis approach. I would have liked, however, some observations regarding choices one might make in these methods that impact the identification of ventral visual areas. For example, they might observe that retinotopic mapping need not extend into the far periphery to define hV4, as the region has a fairly compressed representation of the periphery. Similarly, it would be helpful to mention that different choices can be made in the content of the mapping stimulus that could enhance the responses from hV4. Finally, they might comment upon choices made in EPI vs. spin-echo imaging that might reduce the influence of the transverse venous sinus upon the hV4 functional data, or MR-V imaging techniques that are useful in the identification of this venous structure. These points could also be addressed in a brief paragraph in the discussion.

Second, as the anatomical landmarks are an important guide for the identification of hV4, the authors could comment upon their variability, both within the volumetric space and within a spherical registration space of cortical topology. To my understanding, it is this variability (coupled with variations in structure-function relationships and measurement limitations) that drives the need for individual definition of this region.

Finally, there is a typo in the introduction: the word "calcarine" is unnecessarily capitalized.

Is the rationale for developing the new method (or application) clearly explained? Yes

Is the description of the method technically sound?

Yes

Are sufficient details provided to allow replication of the method development and its use by others? 
Yes

If any results are presented, are all the source data underlying the results available to ensure full reproducibility?

Yes

Are the conclusions about the method and its performance adequately supported by the findings presented in the article?

Yes

Competing Interests: No competing interests were disclosed.

I confirm that I have read this submission and believe that I have an appropriate level of expertise to confirm that it is of an acceptable scientific standard.

Reviewer Report 23 August 2017

https://doi.org/10.5256/f1000research.13389.r25205

(C) 2017 Harvey B. This is an open access peer review report distributed under the terms of the Creative Commons Attribution License, which permits unrestricted use, distribution, and reproduction in any medium, provided the original work is properly cited.

\section{Ben M. Harvey}

Experimental Psychology, Helmholtz Institute, Utrecht University, Utrecht, The Netherlands

Winawer and Witthoft present a methodological paper describing the practical details that allow researchers to localize the human homologue of V4 (hV4) using fMRI visual field mapping. As described in the Abstract and Introduction, it has become very straightforward to define earlier visual field maps (V1-V3), but hV4 presents particular difficulties that prevented researchers even agreeing hV4's location for many years. Studies by Winawer and Witthoft themselves addressed this disagreement. Other researchers in the field have typically learned to localize hV4 after demonstrations of the practical details from experienced colleagues. In most studies, many of these practical details are omitted for brevity, and because parts have been described elsewhere. It is becoming increasingly clear that visual field map definitions beyond V1-V3 are not as consistent as they should be across labs. So, the field will benefit from publication of more standardized, practical protocols like those described here to allow consistent localization of hV4. The approach described here is methodologically very strong and clearly described for an inexperienced researcher.

Major points:

The manuscript describes procedures for drawing V1-3 and hV4. However, the anterior boundary of hV4 is described in relation to VO1 (section 6.2.1), and procedures for drawing VO1 are not described. Furthermore, VO1 is fairly far anterior of hV4, so will often not be covered in a scan volume targeted at V1-3 and hV4. I don't think it is wise to add procedures for drawing V01-2, 
because these procedures rely on their relationship to hV4, making quite a circular description. Therefore, this section would benefit from rewriting without assuming that VO1 has been drawn already, i.e. based on the location of an area of maximum preferred eccentricity in an eccentricity gradient, rather than based on an eccentricity reversal to VO1.

Procedures for drawing hV4 are described clearly, but assume the researcher already has good surface renderings and estimates of each voxel's preferred visual field position. These earlier stages are extensive and complex, but the manuscript describes them only briefly and with reference to previous studies. I don't believe an inexperienced researcher could work through these stages from the brief descriptions in the manuscript, so effectively the manuscript assumes the reader already has considerable experience. To make a more practical, step-by-step description of the complete procedure, a more complete description of normal segmentation and pRF modelling procedures would be valuable. However, I understand if the authors decide against including this, because it would change the focus of the manuscript considerably.

Minor points:

The discussion of differences to expect between subjects is helpful, but the resulting definition of hV4 includes the areas where draining veins introduce artefacts. The manuscript should make clear that subsequent analyses of responses in these areas will be strongly affected by these artefacts. Indeed, for many subsequent analyses these areas give a corrupted view of hV4's responses, and I would certainly exclude these areas from most subsequent analyses. So, the manuscript should make clear that, while these voxels do lie in hV4 anatomically, it is often preferable to exclude them from our definition of hV4.

In Figure 7, the iso-eccentricity line with the highest eccentricity is misleading. In this figure, all voxels with preferred eccentricity above $6^{\circ}$ are labelled in blue. The line running through this blue area therefore links voxels with different eccentricities, all above $6^{\circ}$.

The parahippocampal gyrus is mentioned as a landmark in section 5.2, but not shown on Figure 2 . Please add this to the figure.

In section 6.1, the following phrase is ambiguous: "it may be possible to trace V2 and V3 in concentric ' $V$ ' shapes around V1." This could be taken to mean that each of the dorsal and ventral quarterfields of V2 and V3 is V-shaped (i.e. ending at a point, outside the foveal confluence), when in fact there are bands passing through the foveal confluence (Schira et al, reference 9). Please rewrite this phrase to make it clear that each ' $\mathrm{V}$ ' is a whole hemifield map of V2 or V3.

Is the rationale for developing the new method (or application) clearly explained? Yes

Is the description of the method technically sound?

Yes

Are sufficient details provided to allow replication of the method development and its use by others?

Partly

If any results are presented, are all the source data underlying the results available to 
ensure full reproducibility?

Yes

Are the conclusions about the method and its performance adequately supported by the findings presented in the article?

Yes

Competing Interests: No competing interests were disclosed.

I confirm that I have read this submission and believe that I have an appropriate level of expertise to confirm that it is of an acceptable scientific standard, however I have significant reservations, as outlined above.

The benefits of publishing with F1000Research:

- Your article is published within days, with no editorial bias

- You can publish traditional articles, null/negative results, case reports, data notes and more

- The peer review process is transparent and collaborative

- Your article is indexed in PubMed after passing peer review

- Dedicated customer support at every stage

For pre-submission enquiries, contact research@f1000.com 